\title{
Inferring the selection window in antimicrobial resistance using deep mutational scanning data and biophysics-based fitness models \\ Pouria Dasmeh ${ }^{1,2}$, Anh-Tien Ton ${ }^{1,2}$, Caroline Quach ${ }^{3,4}$, Adrian W.R. Serohijos ${ }^{1,2}$ \\ ${ }^{1}$ Departement de Biochimie, ${ }^{2}$ Centre Robert Cedergren en Bioinformatique et Génomique, Université de Montréal, 2900 Edouard-Montpetit, Montreal, Quebec H3T 1J4, Canada. Département de microbiologie, infectiologie et immunologie, Université de Montréal, ${ }^{4}$ Infection Prevention and Control Unit, Department of Medical Microbiology and Infectious Diseases, CHU Sainte-Justine
}

Correspondence: adrian.serohijos@umontreal.ca

\section{Abstract}

Mutant-selection window (MSW) hypothesis in antimicrobial resistance implies a range

for antimicrobial concentration that promotes selection of single-step resistant mutants.

Since the inception and experimental verification, MSW has been at the forefront of strategies to minimize development of antimicrobial resistance (AR). Setting the upper and lower limits of MSW requires an understanding of the dependence of selection coefficient of arising mutations to antimicrobial concentration. In this work, we employed a biophysics-based and experimentally calibrated fitness model to estimate MSW in the case of Ampicillin and Cefotaxime resistance in E.coli TEM-1 beta lactamase. In line with experimental observations, we show that selection is active at very low levels of

27 antimicrobials. Furthermore, we elucidate the dependence of MSW to catalytic efficiency of mutants, fraction of mutants in the population and discuss the role of

29 population genetic parameters such as population size and mutation rate. Altogether, our analysis and formalism provide a predictive model of MSW with direct implications

31 in the design of dosage strategies. 


\section{Introduction}

36 The gain of resistance to antimicrobials is indeed one of the most challenging threats to

37 public health. An effective approach to contain antimicrobial resistance (AMR) is multi-

38 layered, employing knowledge of the mechanisms for gain of resistance, ecological

39 dynamics of bacterial species and eventually treatment regimens and strategies ${ }^{1}$.

40 Since their first medical use, antimicrobials have been administered at a

41 minimum dosage sufficient to inhibit bacterial growth while keeping hazards minimal.

42 This concentration, termed as minimum inhibitory concentration $(\mathrm{MIC})^{2,3}$, measures

43 susceptibility of bacteria to drugs and is the basis of clinical protocols that compare

44 effectiveness of antimicrobials ${ }^{4}$. The emergence of AMR requires that at least two sub-

45 populations of bacterial species exist, with distinct MICs. The dosage response of

46 bacterial growth to antimicrobials thus defines a concentration window that is above the

47 MIC of wild-type and below the MIC of resistant mutants, i.e., mutant-selection window

48 (MSW) hypothesis ${ }^{5-7}$. The upper limit of this window (i.e., MIC of resistant mutants) is

49 termed as mutant-prevention concentration (MPC) ${ }^{7}$. An effective treatment strategy for

50 AMR sets drug dosage above MPC to inhibit the selection of resistant mutant ${ }^{6}$.

51 Setting the upper and lower limits of MSW is critically important. Obviously, MPC

52 should be high enough to prevent emergence of AMR, but should be the lowest

53 efficacious concentration to minimize side effects. The lower limit, on the other hand, is

54 the minimum selective concentration $(\mathrm{MSC})^{8}$ below which resistance and efficacy are

55 hardly achieved and can be regarded as a safety level for antimicrobial concentration in

56 natural environments ${ }^{9}$. MSC was traditionally assumed to be the MIC of WT strains, but 
57 numerous findings found MSC to be 10- to 100-folds lower and concluded that the

58 selection of resistant mutants is present at sub-MIC concentrations ${ }^{10}$.

59 Emergence of AMR by drug selection pressure is an example of an evolutionary

60 process whose fate is determined by the competition between mutational supply,

61 selection and immigration ${ }^{11}$. Therefore, the rate of acquired resistance depends on the

62 competition between WT and resistant mutants, which in turn depends on several

63 factors such as the mutation rate, selection regime, population size, etc. In the limit of

64 large population size and low mutation rate, selective advantage of the arising mutation

65 becomes the key factor in determining the evolutionary dynamics. In the simplest

66 definition, the selection coefficient (s) of a mutant is the normalized differential fitness

67 between $\mathrm{WT}$ and mutant strains, i.e., $\mathrm{F}_{\mathrm{WT}}$ and $\mathrm{F}_{\text {mut: }}$ :

$$
s=\frac{F_{m u t}-F_{W T}}{F_{W T}}
$$

69 In principle, deleterious, neutral and beneficial nature of mutations is determined by the

70 product of effective population size and selection coefficient, i.e., $\mathrm{N}_{\text {eff }} \times s$ so that $\mathrm{N}_{\text {eff }} \times s<$

$71-1,=1$ and $>1$ corresponds to deleterious, neutral and beneficial mutations,

72 respectively.

73 Over the last decade, considerable efforts have been made to predict protein

74 phenotypes that derive adaptation ${ }^{12,13}$, in particular to drug concentration ${ }^{14,15}$ with the

75 aim of providing an ab initio molecular model for AMR. When the gain of resistance is

76 through de novo mutations in target proteins ${ }^{16}$, the knowledge of genotype to phenotype

77 mapping is crucial in predicting fitness and eventually the selection regime. In these

78 studies, fitness is proportional to bacterial growth, which depends on the metabolic flux 
79 of enzymes targeted by antimicrobials such as dihydrofolate reductase (DHFR) in folate

80 pathway ${ }^{14}$ or competitive inhibition in the case of TEM-1 beta lactamase ${ }^{15,17}$.

81 Given such predictive models of AMR and the resulting fitness landscape from

82 exhaustive mutagenesis experiments, an interesting question is whether these models

83 can be employed to investigate the limits of MSW in AMR. If proved successful, this

84 "bottom-up" approach would result in a predictive model for selective window that will

85 eventually aid in optimizing dosage regimens. This is the major goal of the current

86 work. We will start with a general summary of the predictive model of AR in TEM-1 beta

87 lactamase which relates fitness to the rate of peptidoglycan synthesis and cell wall

88 formation. This rate in turn depends on variety of molecular and cellular factors from

89 permeability of antimicrobials to the catalytic efficiency, $\mathrm{k}_{\text {cat }} / \mathrm{K}_{\mathrm{M}}$, of both penicillin binding

90 proteins (PBPs) and TEM-1 beta lactamase ${ }^{17,18} . \mathrm{MSW}$ is then derived from the fitness

91 equation and the landscape for selection coefficients of arising AMR is explored. We

92 then employ this method to investigate the lower and upper theoretical limits of selective

93 window in AMR and comment on dependence of this limit to factors such as enzyme

94 kinetics and the fraction of resistant mutants in the population.

95

96

97

98

99

100

101

102

103

104

\section{Results}

\section{Fitness model for TEM-1 beta lactamase}

TEM-1 beta lactamase or in general beta lactamases are a family of enzymes that give resistance to beta lactam antibiotics ${ }^{19-22}$. This group of antibiotics which share the common functional group of beta lactam, inhibits formation of the peptidoglycan crosslinks in the bacterial cell wall. The specific mode of action of beta lactam antibiotics is 
105 the competitive binding with DD-transpeptidases, which are collectively known as

106 penicillin binding proteins $(\mathrm{PBP})^{23}$. PBPs are involved in the final step of peptidoglycan

107 synthesis and thus cell wall biogenesis ${ }^{24}$. Resistance to beta lactam antibiotics, by beta

108 lactamases, is achieved through attacking the beta lactam ring of these antibiotics.

109 Therefore, bacterial growth under the concentration of beta lactam antibiotics is

110 proportional to the activity of PBPs.

111 Figure 1 depicts the mechanistic model for the function of TEM-1 beta

112 lactamases within the cell. As in Figure 1A, beta lactam antibiotics diffuse through

113 membrane barrier and once in periplasm, they face either hydrolyses by TEM-1 beta

114 lactamases or diffusion towards their target, PBPs. The fitness of a bacterial cell, i.e.,

115 growth rate, is modeled by the PBPs activity, $v^{\mathrm{PBP}}$ (Figure 1B-C) which is expressed as:

$116 \quad$ Fitness $\propto v^{P B P}=\frac{k_{c a t}^{P B P} \cdot E^{P B P} . C}{\left(1+\frac{[\beta l a c] p}{K_{\beta l a c}^{P B P}}\right) K_{m}^{P B P}+C}$

117 Here, $\mathrm{C}$ and $[\mathrm{Blac}]_{p}$ are the periplasmic concentration of peptidyglycan and beta lactam

118 antibiotic, $k_{c a t}^{P B P}, K_{m}^{P B P}, E^{P B P}$ and $K_{\beta l a c}^{P B P}$ are turnover rate, Michaelis constant,

119 concentration of active PBPs in the periplasm and, dissociation constant of beta lactam

120 antibiotic from PBPs. As assumed in previous works ${ }^{17,18}$, the rate of beta lactam

121 hydrolysis is equal to that of diffusion across periplasm. Hence, one can find the

122 following expression for the concentration of beta lactam antibiotic in the periplasm:

$123[\beta l a c]_{p}=0.5\left([\beta l a c]_{m}-K_{m}^{T E M}-\frac{k_{c a t}^{T E M} \cdot E^{T E M}}{D}+\sqrt{\left(-[\beta l a c]_{m}+K_{m}^{T E M}+\frac{k_{c a t}^{T E M} \cdot E^{T E M}}{D}\right)^{2}+4[\beta l a c]_{m} K_{m}^{T E M}}\right)(\mathrm{Eq}$.

$1243)$ 
125 In Eq. 3, $[\beta l a c]_{m}$ and $D$ are the concentration and the permeability constant for beta

126 lactam antibiotic and $k_{c a t}^{P B P}, K_{m}^{P B P}$ and $E^{P B P}$ are turnover rate, Michaelis constant and the

127 concentration of active beta lactamases in the periplasm.

128 Substituting $[\beta l a c]_{p}$ from Eq. 3 into Eq. 2, selection coefficient (Eq. 1) of an arising

129 mutation can then be calculated as:

$130 \quad S=\frac{\left(\frac{K_{m}^{P B P}}{K_{\beta \operatorname{Plac}}^{P B P}}\right)\left([\beta l a c]_{p}^{W T}-[\beta l a c]_{p}^{\text {mutant }}\right)}{\left(1+\frac{[\text { Blac }]_{p}^{\text {mutant }}}{K_{\beta \text { PIac }}^{P B}}\right) K_{m}^{P B P}+C}$

131 We define the lower and upper limits of MSW, namely MSC and MPC, as the two roots

132 of Eq. 4. We then systematically explore MSC, MPC and MSW as a function of 133 biophysical properties and population genetic parameters. For these analyses, we used

134 three datasets. First, a set of $\mathrm{k}_{\text {cat }}$ and $\mathrm{K}_{\mathrm{M}}$ of 4997 TEM-1 mutants against ampicillin that

135 was obtained by fitting the described fitness model to the relative fitness data from a 136 recent mutational scan $^{17}$. Second, two sets of known kinetic data for TEM-1 variants as

137 tabulated in Table S1-2 for reactions with ampicillin and cefotaxime.

138 We first considered the dependence of selection coefficient to the antimicrobial

139 concentration. Figure 2 shows the average and standard deviation of $s$ for all 4997

140 TEM-1 mutants against Ampicillin. From the figure, mutants become more deleterious

141 i.e., having larger negative selection coefficients, at higher ampicillin concentrations.

142 Figure $2 \mathrm{~B}$ shows the same plot with a finer range of $\mathrm{s}=-0.02$ to 0.02 . The area depicted

143 in gray shows the nearly neutral range (hereafter called nearly-neutral zone)

144 corresponding to $\mid \mathrm{N}_{\text {eff }} \times s / \sim 1$, where the effective population size, $\mathrm{N}_{\text {eff, }}$ is assumed to be

$14510^{7}$. Among 4997 TEM-1 mutants, 32 mutants possess positive selection coefficients at

146 different ranges of antibiotic concentration. As shown in the case of $1173 \mathrm{H}$ mutant, 
147 selection coefficient of beneficial mutations has a quadratic dependence on the

148 antimicrobial concentration and crosses the nearly-neutral zone at a lower and a higher

149 concentration of antibiotic. The lower and higher concentrations correspond to the

150 minimum selective concentration, MSC and, mutant prevention concentration, MPC,

151 respectively (see Methods).

152 We calculated MSC and MPC for TEM-1 beneficial mutations against ampicillin

153 in Figure 3A-B. As shown in Figure 3A, MSC is two-three orders of magnitude lower

154 than MIC of TEM-1 against ampicillin $\left(\sim 1000-4000 \mu \mathrm{g} / \mathrm{ml}^{25-27}\right)$. This shows that

155 selection is active at these very low concentrations in line with previous observations for

156 the prevalence of mutant selection at sub-MIC regimes ${ }^{8,10,28,29}$. There is an inverse

157 exponential relationship between MPC and MSC as shown in Figure 3C. Beneficial

158 mutations with higher selective advantages at lower antimicrobial concentrations would

159 indeed require higher antimicrobial concentrations for prevention. The maximum MPC

160 belonged to P27E mutant $\left(\sim 10^{6} \mu \mathrm{g} / \mathrm{ml}\right)$. Notably, mutations in residue 173 that are

161 observed in laboratory evolution of TEM-1 $1^{30,31}$ and in clinical isolates ${ }^{32}$ are among

162 beneficial mutations.

163 Next, we looked at the individual $s$ curves for TEM-1 mutants against ampicillin.

164 As shown in Figure 4A, selection coefficients are sigmoidal in antimicrobial

165 concentrations with two interesting features: I) selection coefficients are clustered and

166 non-randomly distributed at different concentrations and II) mutations do not preserve

167 their selective rank at all antimicrobial concentrations. Simply put a high fit mutant to

168 lower concentrations of ampicillin (compared to all other mutants) would not retain the 
169 same fitness rank at higher concentrations. Below, we discuss these two features in 170 detail.

171 The clustering of selection coefficients of several mutations implies that s values 172 are non-uniformly distributed. To see the pattern of clustering, we compared the 173 distribution of distances between selection coefficients with that of an equidistance 174 distribution using Kolmogorov-smirnov test. Selection coefficients at all antimicrobial 175 concentrations were significantly non-equidistant ( $p$-value $\left.<10^{-16}\right)$. Then, we looked at 176 the optimum number of clusters and the centroids as shown in Figure 4C-D for each 177 ampicillin concentration up to $10000 \mu \mathrm{g} / \mathrm{ml}$ ( two folds higher than MIC). Several 178 features of these plots are interesting. First, at low antimicrobial concentrations most 179 selection coefficients are within the $8^{\text {th }}$ and $9^{\text {th }}$ clusters being highly deleterious 180 (compare upper left dashed squares in Figures $4 \mathrm{C}$ and $4 \mathrm{C}$ ). This trend is reversed at 181 high concentrations. As shown in bottom-right dashed squares in Figures 4C-D, at high 182 concentrations of antimicrobials, almost half of mutations have neutral or negligible 183 fitness costs. This behavior is in contrast with the average behavior shown in Figure $2 \mathrm{~A}$ 184 showing a rich heterogeneity in selective advantage/disadvantage of mutant clusters at 185 different antibiotic concentrations. Interestingly, a sudden overpopulation of mutants 186 ( 2/5 of all mutants) in lower more neutral clusters occurs around MIC, shown with a 187 black arrow in Figure 4C.

188 Another interesting feature of Figure $4 \mathrm{~A}$ is that s-curves cross each other at different 189 antimicrobial concentrations. This implies that the rank of different mutants is not 190 preserved at all antimicrobial concentrations. To illustrate this observation further, we 191 plotted selection coefficients of two mutants, L152C and G251R, in black and gray in 
192 Figure $5 \mathrm{~A}$. The corresponding values for $\mathrm{V}_{\max }$ and $\mathrm{K}_{\mathrm{M}}$ of both mutants are shown

193 accordingly. From the figure, at low antimicrobial concentrations, the mutant with lower

194 half-saturation limit (L152C) has a higher selective advantage despite having 16\% less

195 turnover rate. The more efficient G251R mutant gains a higher fitness once

196 antimicrobial concentration reaches the corresponding half-saturation limit.

197 The lack of rank-preservation at different antibiotic concentrations means that a 198 mutant that confers resistance at one antibiotic concentration would not necessarily do

199 so at a different concentration. This adds another level of impairment for predictability of

200 antimicrobial resistance in addition to stochastic rise and fixation of resistant mutations.

201 To check the rank of mutants at different antimicrobial concentrations systematically, we

202 compared the rank of all TEM-1 mutants at 9 antimicrobial concentrations 1000 to 9000

$203 \mu \mathrm{g} / \mathrm{ml}$ to the ranks at $1 \mu \mathrm{g} / \mathrm{ml}$. As shown in Figure 5B, the original rank is lost at all

204 higher concentrations. In fact, the correlation between the rank of mutants among all

205 concentrations although significant is very weak $\left(R \sim 0.01\right.$, $p$-value $<10^{-3}$; see Figure $S 1$ -

206 2). We also checked the preservation of ranks at ampicillin concentrations below 1000

$207 \mu \mathrm{g} / \mathrm{ml}$. As shown in Figure $5 \mathrm{C}$, the maximum correlation is $\sim 0.35$ which decays to $\sim 0.05$

208 as the antimicrobial concentration increases.

209 To explore the dependence of MSW to enzyme kinetics, we plotted $\mathrm{V}_{\max }$ and $\mathrm{K}_{\mathrm{M}}$

210 of TEM-1 beneficial mutations. Deleterious and neutral mutations are shown in gray and

211 beneficial mutations in black. From the figure, beneficial mutations have maximized $\mathrm{V}_{\max }$

212 and minimized $\mathrm{K}_{M}$ leading to maximized $\mathrm{V}_{\max } / \mathrm{K}_{\mathrm{M}}$ ratio (see the fitted dashed line to all

213 beneficial mutations in Figure 6A). We then plotted MSC and MPC versus $V_{\text {max }} / K_{M}$ of all

214 beneficial mutants of TEM-1 (Figure 6B-C). From the figure, mutants with a higher 
215 catalytic efficiency have lower MSC and higher MPC giving rise to an increased MSW.

216 We thus conclude that $\mathrm{V}_{\text {max }} / \mathrm{K}_{\mathrm{M}}$ ratio is a strong determinant of MSC and MPC in the

217 case of TEM-1 and ampicillin resistance.

218 To what extend the selection regime and the concentration-dependence of

219 selection coefficient discussed in Figure 2 are common to other beta-lactam

220 antimicrobials? To answer this question, we sought to analyze fitness effects of arising

221 mutations in TEM-1 against cefotaxime. Resistance to cefotaxime is widely reported for

222 TEM-1 and the extended-spectrum beta lactamases (ESBLs). Figure S2 shows the

223 selection coefficient curves for TEM-1 mutants and natural variants where kinetic data

224 for reaction with cefotaxime were available (Table S2). Evidently, a major difference

225 with the case of ampicillin is the degree to which mutants are beneficial. While almost all

226 TEM-1 mutants ( 99\% in the DMS data set) and all natural variants (Figure S3 and

227 Table S2) were deleterious at all ampicillin concentrations, kinetic data against

228 cefotaxime shows a substantially higher fraction of beneficial mutants (12 out of 16

229 mutants, Figure S4). From the figure, all ESBLs and TEM-1 mutants share the initial

230 rise in selection coefficient within the few hundred $\mu \mathrm{g} / \mathrm{ml}$ of cefotaxime. Note that MIC of

231 cefotaxime is $\sim 6 \mu \mathrm{M}$. The strongly beneficial nature of mutants in TEM-1 against

232 cefotaxime causes MSC to be $\sim 1 \mu \mathrm{g} / \mathrm{ml}$ for all mutants. MPC is never reached within the

233 concentration range studied here, i.e., up to $10^{6} \mu \mathrm{g} / \mathrm{ml}$. As shown in Figure S5, the

234 maximum $s$ in cefotaxime dataset reaches is almost two orders of magnitude higher

235 than the selection coefficient of the most beneficial TEM-1 mutant against ampicillin. 


\section{Discussion}

239 The knowledge of antimicrobial concentration at which resistance is prevented is crucial

240 for designing dosage strategies. As we showed in this work, and for the specific case of

241 TEM-1 beta lactamase against ampicillin and cefotaxime, a direct determination of MSC

242 and MPC is feasible if the fitness landscape of enzymes conferring antimicrobial

243 resistance, such as TEM-1 beta lactamase is known. As we showed in this work, both

244 MSC and MPC depend on catalytic efficiency of mutants, i.e., the $V_{\max } / K_{M}$ ratio in the

245 case of ampicillin. However, for strongly beneficial mutations, as observed in the case of

246 cefotaxime, MSC is essentially zero and MPC fall beyond the range of biologically

247 relevant concentrations. One would thus expect that resistance to cefotaxime is much

248 more prevalent than to ampicillin. Therefore, it is essential to estimate not only the

249 fraction of beneficial mutations but also the magnitude of fitness effects, i.e., the full

250 distribution of fitness effects (DFE). We thus propose that DFE obtained from deep

251 mutational scanning combined with fitness models, either biophysics-based as in this

252 work or phenomenological ${ }^{33}$, has a great potential to estimate the spectrum of

253 resistance.

254 In all calculations in this work, we assumed that both WT and mutants have

255 equal initial finesses. This assumption is biologically unrealistic as resistant mutants are

256 shown to be enriched in the population from very low fractions of the order of $10^{-4} 29$. To

257 check for this effect, we used the fraction of mutants in the population as a scaling

258 factor for fitness in Equation 2 (see Methods) and plotted selection coefficients versus

259 ampicillin for the case of $1173 \mathrm{H}$ mutant in Figure 7B. From the figure, MSW is essentially

260 zero for the same beneficial mutation but with negligible fractions in the population. 
261 Therefore, figures 7B entails a window of opportunity for containment of AMR if 262 antibiotics are administered at the very onset of emergence of resistance. An exact

263 profiling of both type and fraction of resistant mutants is necessary for this purpose.

264 Two factors, namely bacterial population size and immune response, influence

265 the near-neutrality zone and potentially influence MSW and the fraction of beneficial

266 mutations. First, from classical population genetics, population size scales up fixation of

267 beneficial mutations. Since selection is stringer at higher population sizes, slightly

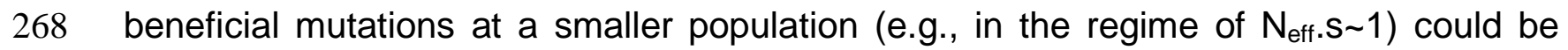

269 fixated with a higher rate at the larger population. Therefore, higher population sizes

270 decrease the width of near-neutrality zone and gives rise to lower MSC, higher MPC

271 and a wider MSW.

272 Second, in the absence of immune defense as in laboratory settings, the near-

273 neutrality zone defines the lowest limit of selection. However, a major difference

274 between these settings and the clinical settings as in the patient body is the presence of

275 immune defense. In fact, the first response to bacterial infections is through the immune

276 system. Therefore, one expects that the real selection limit is extended above the near-

277 neutrality zone and to the limit below which infection is contained by the immune

278 defense. This factor narrows down the MSW and thus the number of resistant

279 conferring mutations is further decreased.

280 We summarize the role of confounding factors on MSW as studied in this work in

281 Figure 7B. First, any factor that increases selective advantage of mutants such as a

282 higher catalytic efficiency widens MSW. We provided an equation for the relationship

283 between MSC and MPC to the $\mathrm{V}_{\max } / \mathrm{K}_{\mathrm{M}}$ ratio and expect that such relationships exist for 
284 other antimicrobials and target enzymes. Second, MSW is substantially narrowed when

285 the fraction of resistant mutations is negligible in the population. Third, lower population

286 sizes or higher immune defense extend the near-neutrality zone and thus narrow down

287 MSW. We anticipate that optimization of MSW with respect to each of these factors

288 would provide a powerful strategy for rational design of dosage strategy in the treatment

289 of antimicrobial resistance.

\section{Materials and method:}

\section{Fitness function}

296 Determination of both upper and lower limits to MSW in our approach requires proper

297 definition of fitness of an organism. Following the original work of Zimmerman and 298 Rosselet $^{18}$ and the following theoretical works ${ }^{17,34,35}$, we assumed that beta lactam 299 antimicrobials passively diffuse through the outer membranes ${ }^{36,37}$. Once in the

300 periplasm, the antimicrobial either get hydrolyzed by beta lactamase or diffuses to the

301 target PBP where competitively inhibits formation of peptidoglycan by interacting with

302 penicillin binding proteins (Figure $1 \mathrm{~A}$ ). The rate equations for beta lactam antibiotics and 303 peptidoglycan synthesis can be written as

$304 \frac{d}{d t}[\beta l a c]_{p}=D\left([\beta l a c]_{m}-[\beta l a c]_{p}\right)-\frac{k_{c a t}^{\text {Blactamase }}[\text { Blactamase }][\beta l a c]_{p}}{K_{M}^{\text {Blactamase }}+[\beta \text { lac }]_{p}}=0$

$305 \frac{d}{d t}[$ PepGly $]=\frac{k_{c a t}^{P B P}[P B P][\text { PepGly }]}{\left(1+\frac{[\beta l a c] p}{K_{\beta l a c}^{P B P}}\right) K_{M}^{P B P}+[\text { PepGly }]} \equiv$ Fitness 
As described earlier, the rate of beta lactam diffusion in the periplasm is assumed to be equal to that of hydrolysis by beta lactamase ${ }^{17,18}$. Using fitness from Eq. 6 , the relative fitness $(\hat{F})$ of each mutant to WT TEM-1 is then expressed as:

309

$\hat{F}=\frac{A}{1+B[\beta l a c]_{p}^{m u t}}-\frac{A}{1+B[\beta l a c]_{p}^{W T}}$

where $A=k_{\text {cat }}^{P B P}[P B P][P e p G l y]$ and $B=\frac{K_{M}^{P B P}}{K_{\beta l a c}^{P B P}\left(K_{M}^{P B P}+[\text { PepGly] })\right.}$. Stiffler et al. ${ }^{17}$ fitted equation

7 to relative fitness obtained from deep mutational scanning data and estimated both $A$

312 and $\mathrm{B}$ constants and $\mathrm{K}_{\mathrm{cat}}$ and $\mathrm{K}_{\mathrm{M}}$ of 4997 TEM-1 mutants using a monte carlo simulated

313 annealing approach.

314

315

Table 1. Parameters used in the fitness function with the corresponding values.

\begin{tabular}{|c|c|c|c|}
\hline Parameter & Definition & Value (unit) & References \\
\hline$K_{\text {cat }}{ }^{\text {PBP }}$ & Enzyme turnover rate of PBPs & $\sim 0.05\left(\mathrm{~s}^{-1}\right)^{\mathrm{a}}$ & 38,39 \\
\hline$K_{\mathrm{M}}{ }^{\text {PBP }}$ & Michaelis constant of PBPs & $\sim 83 \mathrm{mM}$ & 38,39 \\
\hline$E_{\mathrm{PBP}}$ & Concentration of folded and active PBPs & $0.12-4 \mu \mathrm{M}$ & Calculated $^{b}$ \\
\hline$E_{\mathrm{PG}}$ & Periplasmic concentration of peptidoglycan & $\sim 11 \mu \mathrm{M}$ & 40 \\
\hline$K_{\text {blac }}{ }^{\text {PBP }}$ & Dissociation constant of beta-lactam from & $200-1600 \mu \mathrm{M}^{-1}$ & 41 \\
\hline$k_{\text {cat }}{ }^{\text {TEM-1 }}$ & EnPs & $\sim 1-5000(1 / \mathrm{s})$ & $25,26,42-50$ \\
\hline$K_{\mathrm{M}}{ }^{\text {TEM-1 }}$ & Michaelis constant of TEM-1 & $\sim 4-3500(1 / \mathrm{s})$ & $25,26,42-50$ \\
\hline$E_{\mathrm{TEM}-1}$ & Concentration of folded and active TEM-1 & $\sim 3-4 \mu \mathrm{M}$ & 17 \\
\hline$D$ & permeability parameter for beta-lactam & $\sim 0.002-0.005$ & 17 \\
\hline
\end{tabular}

a: For values in ranges, the average value is used in the model. b: See supplementary information for details.

\section{References}

1. Lee, C.-R., Cho, I.H., Jeong, B.C. \& Lee, S.H. Strategies to minimize antibiotic resistance. International journal of environmental research and public health 10, 4274-4305 (2013).

2. Birkhaug, K.E. Phenyl-mercuric-nitrate. The Journal of Infectious Diseases, 250-261 (1933). 
3. Garrod, L.P. The effect of bacterial numbers on minimum bacteriostatic concentrations. Journal of Infectious Diseases 57, 247-251 (1935).

4. Schwalbe, R., Steele-Moore, L. \& Goodwin, A.C. Antimicrobial susceptibility testing protocols, (Crc Press, 2007).

5. Drlica, K. The mutant selection window and antimicrobial resistance. Journal of Antimicrobial Chemotherapy 52, 11-17 (2003).

6. Drlica, K. \& Zhao, X. Mutant selection window hypothesis updated. Clinical infectious diseases 44, 681-688 (2007).

7. Dong, Y., Zhao, X., Domagala, J. \& Drlica, K. Effect of fluoroquinolone concentration on selection of resistant mutants of Mycobacterium bovis BCG andStaphylococcus aureus. Antimicrobial agents and chemotherapy 43, 1756-1758 (1999).

8. Liu, A. et al. Selective advantage of resistant strains at trace levels of antibiotics: a simple and ultrasensitive color test for detection of antibiotics and genotoxic agents. Antimicrobial agents and chemotherapy 55, 1204-1210 (2011).

9. Baquero, F., Martínez, J.-L. \& Cantón, R. Antibiotics and antibiotic resistance in water environments. Current opinion in biotechnology 19, 260-265 (2008).

10. Andersson, D.I. \& Hughes, D. Microbiological effects of sublethal levels of antibiotics. Nature Reviews Microbiology 12, 465-478 (2014).

11. zur Wiesch, P.A., Kouyos, R., Engelstädter, J., Regoes, R.R. \& Bonhoeffer, S. Population biological principles of drug-resistance evolution in infectious diseases. The Lancet infectious diseases 11, 236-247 (2011).

12. Dean, A.M. \& Thornton, J.W. Mechanistic approaches to the study of evolution: the functional synthesis. Nature Reviews Genetics 8, 675-688 (2007).

13. Harms, M.J. \& Thornton, J.W. Evolutionary biochemistry: revealing the historical and physical causes of protein properties. Nature Reviews Genetics 14, 559-571 (2013).

14. Rodrigues, J.V. et al. Biophysical principles predict fitness landscapes of drug resistance. Proceedings of the National Academy of Sciences, 201601441 (2016).

15. Knies, J.L., Cai, F. \& Weinreich, D.M. Enzyme efficiency but not thermostability drives cefotaxime resistance evolution in TEM-1 $\beta$-lactamase. Molecular biology and evolution 34, 1040-1054 (2017).

16. Woodford, N. \& Ellington, M.J. The emergence of antibiotic resistance by mutation. Clinical Microbiology and Infection 13, 5-18 (2007).

17. Stiffler, M.A., Hekstra, D.R. \& Ranganathan, R. Evolvability as a function of purifying selection in TEM-1 $\beta$-lactamase. Cell 160, 882-892 (2015).

18. Zimmermann, W. \& Rosselet, A. Function of the outer membrane of Escherichia coli as a permeability barrier to beta-lactam antibiotics. Antimicrobial Agents and Chemotherapy 12, 368-372 (1977).

19. Livermore, D.M. beta-Lactamases in laboratory and clinical resistance. Clinical microbiology reviews 8, 557-584 (1995).

20. Philippon, A., Labia, R. \& Jacoby, G. Extended-spectrum beta-lactamases. Antimicrobial agents and chemotherapy 33, 1131 (1989).

21. Jacoby, G.A. \& Medeiros, A.A. More extended-spectrum beta-lactamases. Antimicrobial Agents and Chemotherapy 35, 1697 (1991). 
22. Bradford, P. Extended-spectrum betalactamases in the 21st century: characterization, epidemiology, and detection of this important resistance threat. ClinMicrobiol Rev 2001; 14: 933-51. B, III (1999).

23. Hartman, B.J. \& Tomasz, A. Low-affinity penicillin-binding protein associated with betalactam resistance in Staphylococcus aureus. Journal of Bacteriology 158, 513-516 (1984).

24. Sauvage, E., Kerff, F., Terrak, M., Ayala, J.A. \& Charlier, P. The penicillin-binding proteins: structure and role in peptidoglycan biosynthesis. FEMS microbiology reviews 32, 234258 (2008).

25. Huang, W., Le, Q.-Q., LaRocco, M. \& Palzkill, T. Effect of threonine-to-methionine substitution at position 265 on structure and function of TEM-1 beta-lactamase. Antimicrobial agents and chemotherapy 38, 2266-2269 (1994).

26. Marciano, D.C., Brown, N.G. \& Palzkill, T. Analysis of the plasticity of location of the Arg244 positive charge within the active site of the TEM-1 $\beta$-lactamase. Protein Science 18, 2080-2089 (2009).

27. Salverda, M.L. et al. Initial mutations direct alternative pathways of protein evolution. PLoS genetics 7, e1001321 (2011).

28. Zhanel, G.G., Mayer, M., Laing, N. \& Adam, H.J. Mutant prevention concentrations of levofloxacin alone and in combination with azithromycin, ceftazidime, colistin (Polymyxin E), meropenem, piperacillin-tazobactam, and tobramycin against Pseudomonas aeruginosa. Antimicrobial agents and chemotherapy 50, 2228-2230 (2006).

29. Gullberg, E. et al. Selection of resistant bacteria at very low antibiotic concentrations. PLoS pathogens 7, e1002158 (2011).

30. Barlow, M. \& Hall, B.G. Predicting evolutionary potential: in vitro evolution accurately reproduces natural evolution of the TEM $\beta$-lactamase. Genetics 160, 823-832 (2002).

31. Barlow, M. \& Hall, B.G. Experimental prediction of the natural evolution of antibiotic resistance. Genetics 163, 1237-1241 (2003).

32. Zarnayová, M. et al. Survey of Enterobacteriaceae producing extended-spectrum $\beta$ lactamases in a Slovak hospital: dominance of SHV-2a and characterization of TEM-132. Antimicrobial agents and chemotherapy 49, 3066-3069 (2005).

33. Levison, M.E. \& Levison, J.H. Pharmacokinetics and pharmacodynamics of antibacterial agents. Infectious disease clinics of North America 23, 791-815 (2009).

34. Nikaido, H. \& Normark, S. Sensitivity of Escherichia coli to various $\beta$-lactams is determined by the interplay of outer membrane permeability and degradation by periplasmic $\beta$-lactamases: a quantitative predictive treatment. Molecular microbiology 1, 29-36 (1987).

35. Lakaye, B., Dubus, A., Joris, B. \& Frère, J.-M. Method for estimation of low outer membrane permeability to $\beta$-lactam antibiotics. Antimicrobial agents and chemotherapy 46, 2901-2907 (2002).

36. Richmond, M. \& Sykes, R. The $\beta$-lactamases of gram-negative bacteria and their possible physiological role. Advances in microbial physiology 9, 31-88 (1973).

37. Nikaido, H. Outer membrane of Salmonella typhimurium: transmembrane diffusion of some hydrophobic substances. Biochimica et Biophysica Acta (BBA)-Biomembranes 433, 118-132 (1976). 
38. Zhang, W., Shi, Q., Meroueh, S.O., Vakulenko, S.B. \& Mobashery, S. Catalytic mechanism of penicillin-binding protein 5 of Escherichia coli. Biochemistry 46, 10113-10121 (2007).

39. Van der Linden, M., De Haan, L., Dideberg, O. \& Keck, W. Site-directed mutagenesis of proposed active-site residues of penicillin-binding protein 5 from Escherichia coli. Biochemical Journal 303, 357-362 (1994).

40. Wientjes, F., Woldringh, C. \& Nanninga, N. Amount of peptidoglycan in cell walls of gram-negative bacteria. Journal of bacteriology 173, 7684-7691 (1991).

41. Fuda, C., Suvorov, M., Vakulenko, S.B. \& Mobashery, S. The basis for resistance to $\beta$ lactam antibiotics by penicillin-binding protein 2a of methicillin-resistant Staphylococcus aureus. Journal of Biological Chemistry 279, 40802-40806 (2004).

42. Cantu, C., Huang, W. \& Palzkill, T. Cephalosporin substrate specificity determinants of TEM-1 $\beta$-lactamase. Journal of Biological Chemistry 272, 29144-29150 (1997).

43. LENFANT, F. et al. Site-directed mutagenesis of $\beta$-lactamase TEM-1. The FEBS Journal 217, 939-946 (1993).

44. Imtiaz, U., Manavathu, E.K., Lerner, S. \& Mobashery, S. Critical hydrogen bonding by serine 235 for cephalosporinase activity of TEM-1 beta-lactamase. Antimicrobial agents and chemotherapy 37, 2438-2442 (1993).

45. Gibson, R., Christensen, H. \& Waley, S. Site-directed mutagenesis of $\beta$-lactamase I. Single and double mutants of Glu-166 and Lys-73. Biochemical journal 272, 613-619 (1990).

46. Majiduddin, F.K. \& Palzkill, T. An analysis of why highly similar enzymes evolve differently. Genetics 163, 457-466 (2003).

47. Raquet, X. et al. TEM $\beta$-lactamase mutants hydrolysing third-generation cephalosporins: a kinetic and molecular modelling analysis. Journal of molecular biology 244, 625-639 (1994).

48. Salverda, M.L., De Visser, J.A.G. \& Barlow, M. Natural evolution of TEM-1 $\beta$-lactamase: experimental reconstruction and clinical relevance. FEMS microbiology reviews 34, 1015-1036 (2010).

49. Sideraki, V., Huang, W., Palzkill, T. \& Gilbert, H.F. A secondary drug resistance mutation of TEM-1 $\beta$-lactamase that suppresses misfolding and aggregation. Proceedings of the National Academy of Sciences 98, 283-288 (2001).

50. Thomas, V.L. et al. Structural consequences of the inhibitor-resistant Ser130Gly substitution in TEM $\beta$-lactamase. Biochemistry 44, 9330-9338 (2005). 

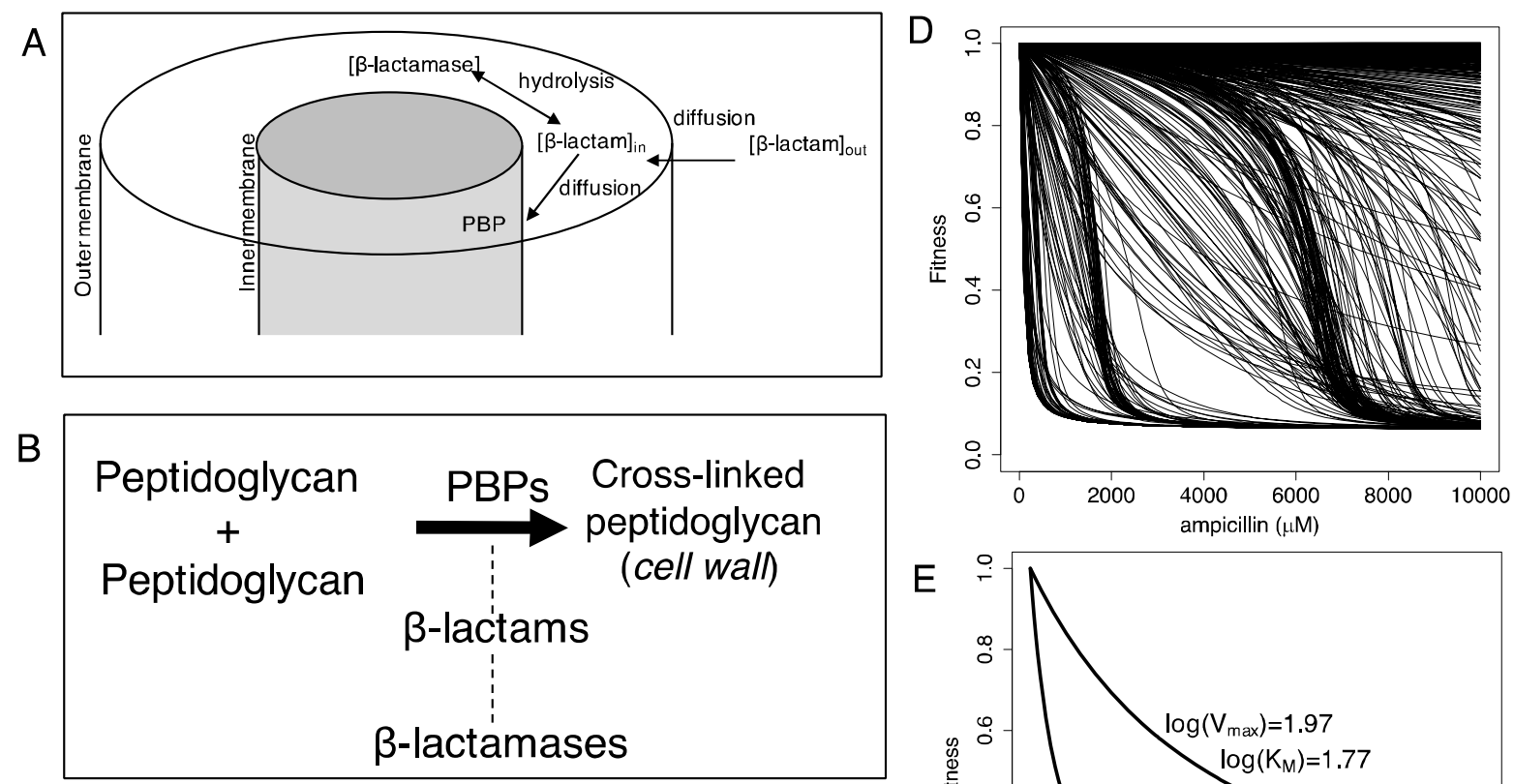

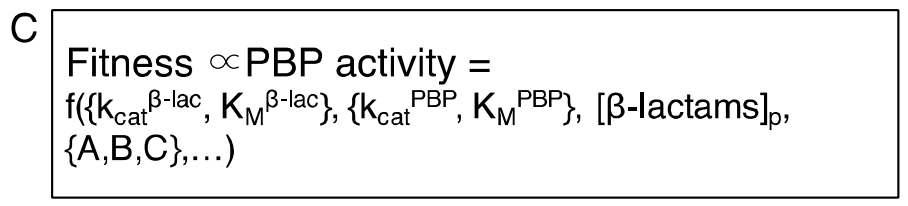

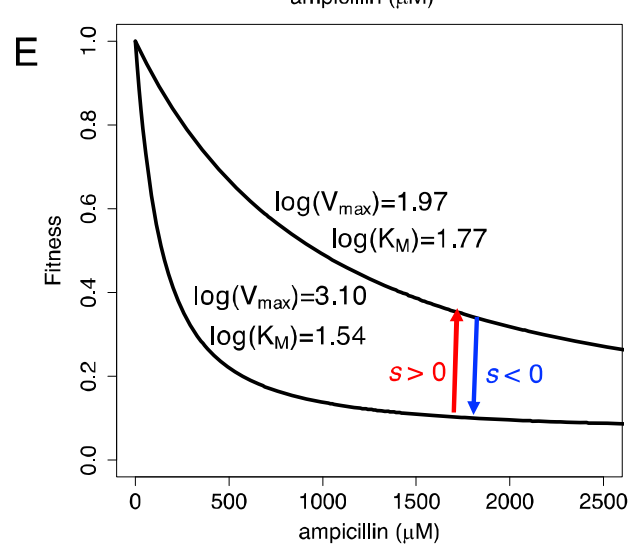

Figure 1. Scheme of the fitness model and the corresponding dosage dependence profile for selection coefficient. A) Beta lactam antimicrobials diffuses through the outer membrane and faces either diffusion to their targets, penicillin binding proteins, PBPs or hydrolysis by beta lactamases. B) Fitness is proportional to the rate of peptidoglycan formation and the activity of penicillin binding proteins. Beta lactams involve in competitive inhibition of PBPs. The resulting fitness function is a function of I) catalytic efficiency of beta lactamases, catalytic efficiency of PBPs, concentration of beta lactams in the periplasm and cellular constants such a permeability of beta lactam antimicrobials. D) Using the functionality of growth rate to different molecular and cellular constants, fitness of different mutants can be expressed as a function of antimicrobial concentration. E) Selection coefficient of an arising mutation can be expressed as a function of antimicrobial concentration depending on the fitness curves of WT and resistant mutants. If mutant is more fit than WT (e.g., has a higher kinetic efficiency-the upper curve), 


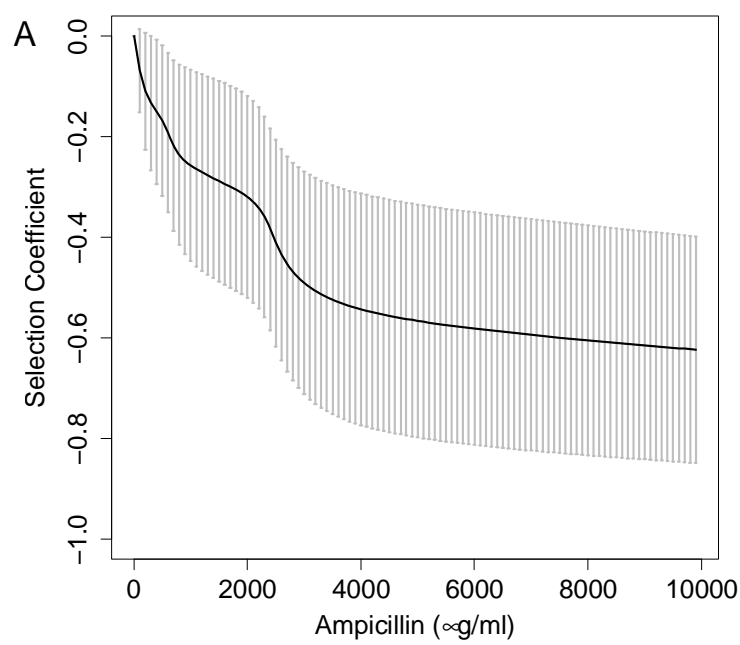
selection coefficients fall in the nearly-neutral zone.
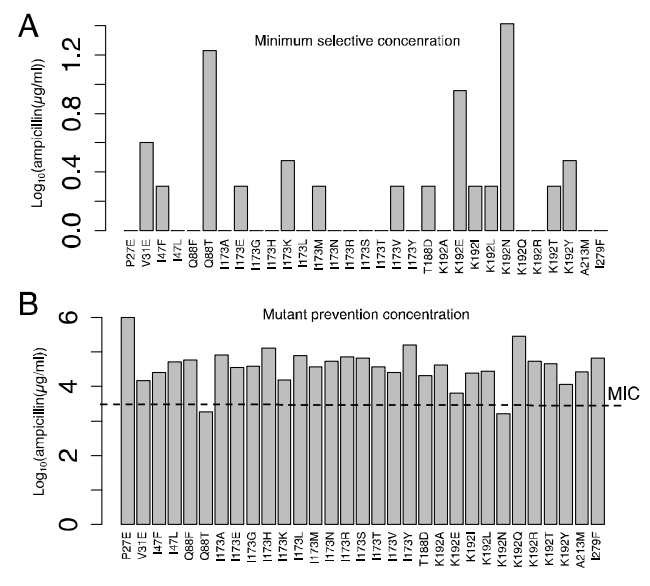
concentration, MIC, of WT TEM-1.

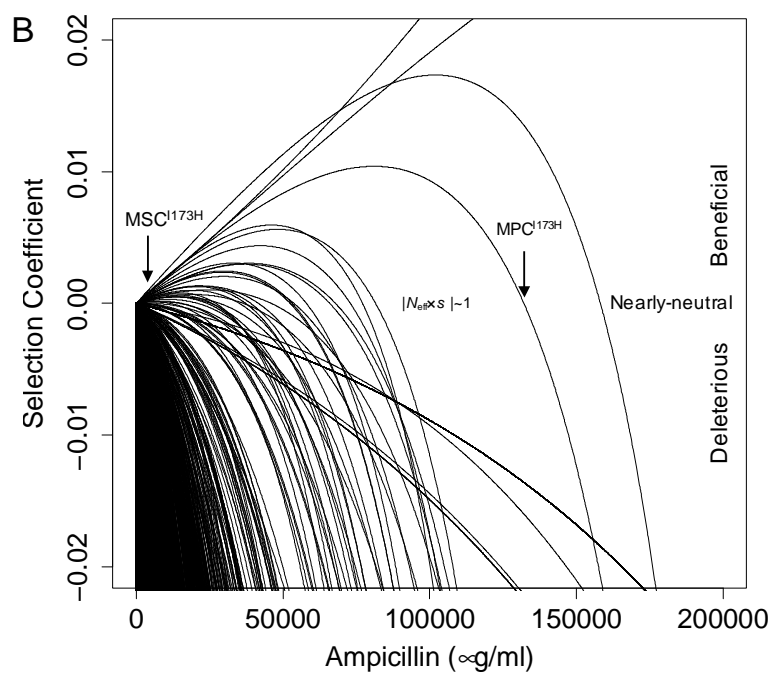

Figure 2. Selection coefficient of TEM-1 mutants versus ampicillin concentration. A) Average (black line) and standard deviation (gray bars) of selection coefficient of 4997 mutants of TEM- 1 versus ampicillin concentration. B) Beneficial mutations show a quadratic concave dependence to ampicillin concentrations. The gray area is the zone at which the mutations have nearly-neutral effects on fitness. Above and below this zone, mutations are either beneficial or deleterious. Minimum selective concentration (MSC) and mutant prevention concentration (MPC) are the concentrations at which

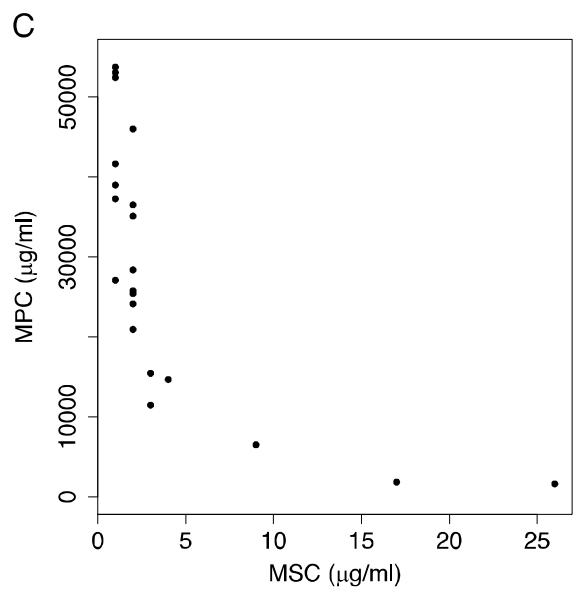

Figure 3. Lower bound, minimum selective concentration, MSC, and upper bound, mutant prevention concentration, MPC of mutant selection window. A) MSC B) MPC and C) MPC versus MSC of beneficial mutants in TEM-1 with ampicillin. The dashed line shows minimum inhibitory 
B
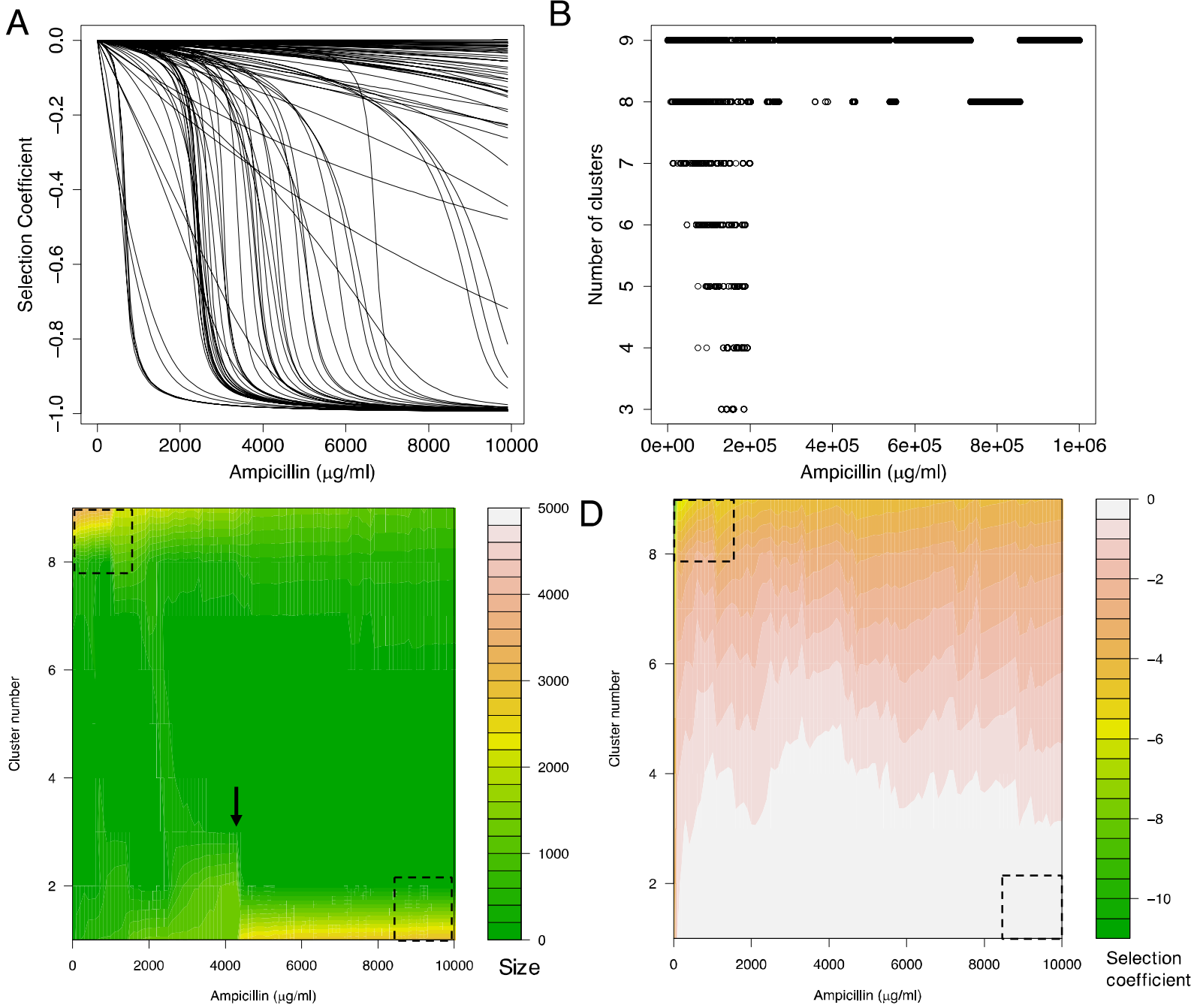

Figure 4. Significant heterogeneity of selection coefficients at different ampicillin concentrations. A) The selection coefficient curves (s-curves) for a set of 100 mutants. B) Clustering of s-curves at different ampicillin concentrations. Up to 9 unique clusters are detected with different sizes. C) Size and D) centroid of each cluster at different antimicrobial concentrations. 

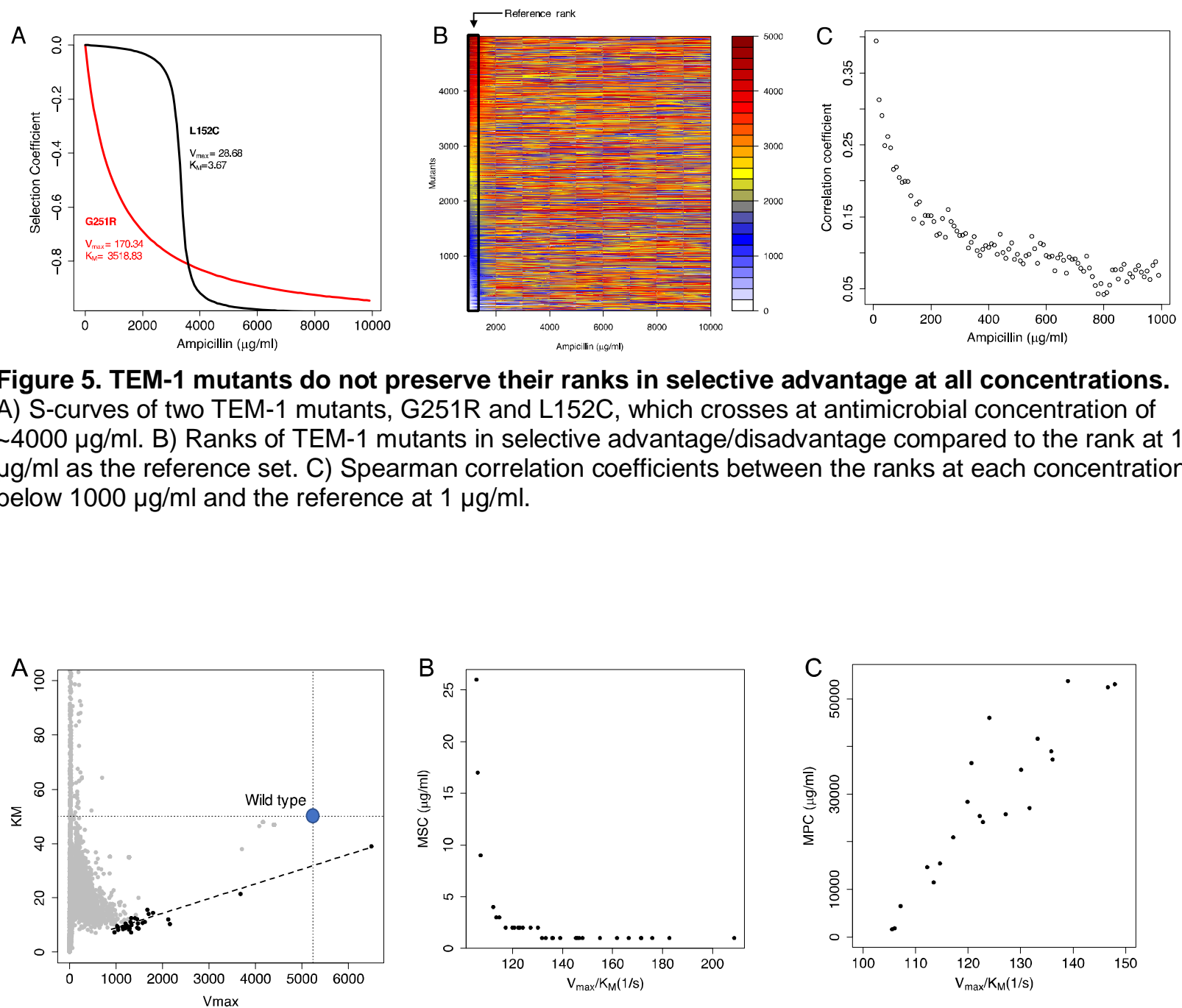

Figure 5. TEM-1 mutants do not preserve their ranks in selective advantage at all concentrations. A) S-curves of two TEM-1 mutants, G251R and L152C, which crosses at antimicrobial concentration of $\sim 4000 \mu \mathrm{g} / \mathrm{ml}$. B) Ranks of TEM-1 mutants in selective advantage/disadvantage compared to the rank at 1 $\mu \mathrm{g} / \mathrm{ml}$ as the reference set. C) Spearman correlation coefficients between the ranks at each concentration below $1000 \mu \mathrm{g} / \mathrm{ml}$ and the reference at $1 \mu \mathrm{g} / \mathrm{ml}$.

Figure 6. MSC and MPC of TEM-1 beneficial mutations as a function of catalytic efficiency. A) $K_{M}$ versus $V_{\max }$ for all (shown in gray) and beneficial (shown in black) TEM-1 mutants. B) MSC and C) MPC of beneficial mutants versus catalytic efficiency.
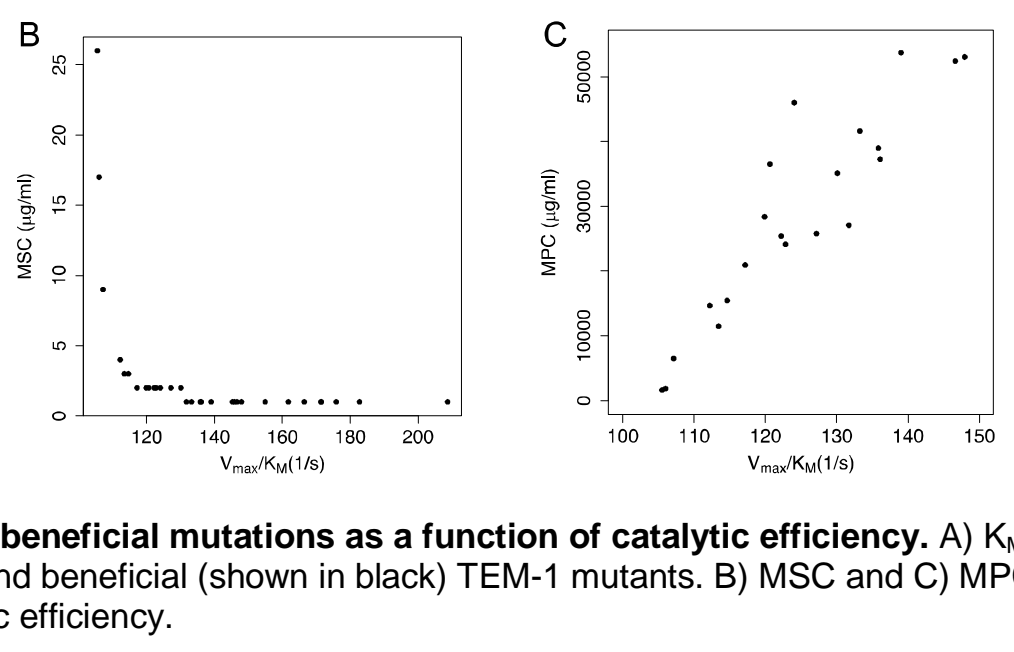
A

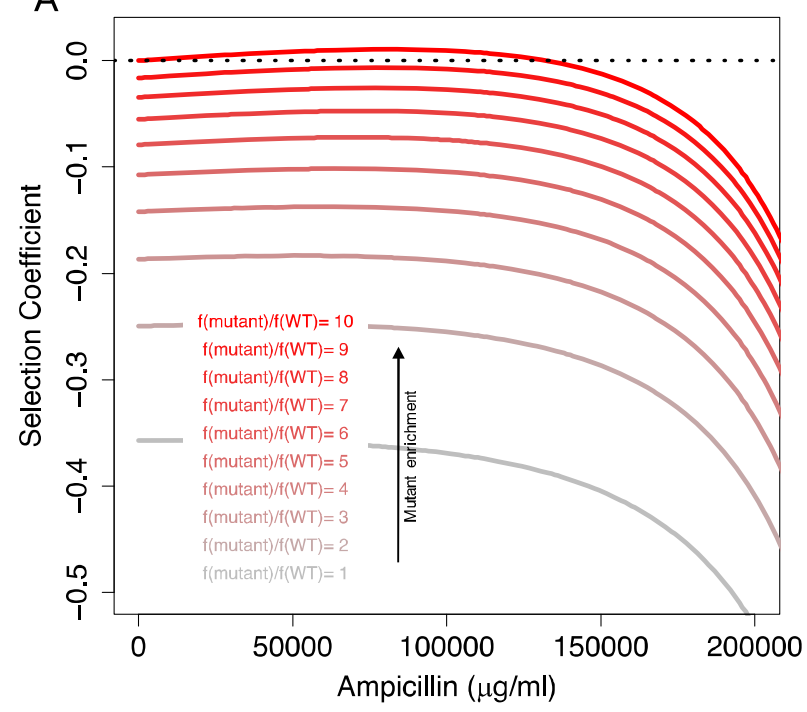

\section{B}

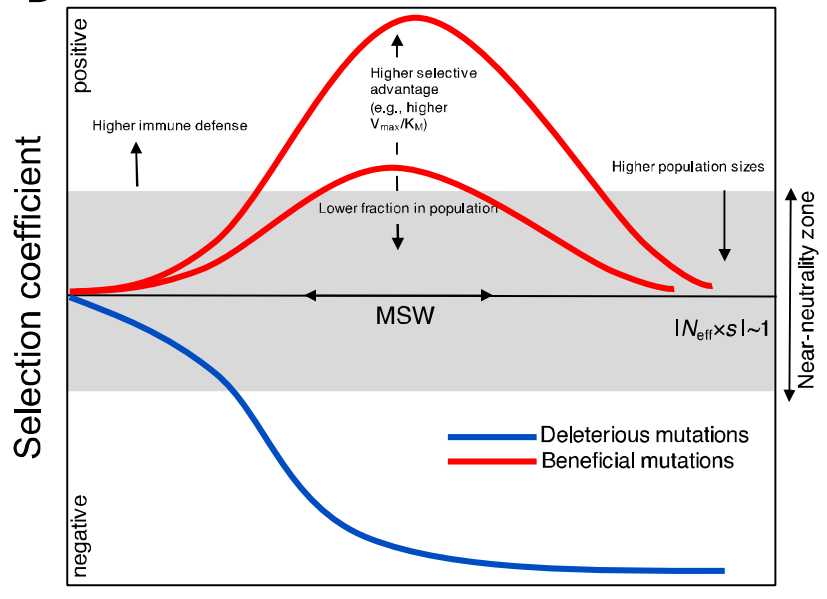

Antibiotic concentration

Figure 7. A) Selection coefficient of a beneficial TEM-1 mutant $(\mathrm{I173H})$ versus ampicillin concentration as a function of mutant fraction in the population. B) The schematic influence of confounding factors such as enzymatic efficiency and fraction in the population on MSW. 